\section{Riboswitch-dependent gene regulation and its evolution in the plant kingdom}

\author{
Samuel Bocobza, ${ }^{1,2}$ Avital Adato, ${ }^{1}$ Tali Mandel, ${ }^{1}$ \\ Michal Shapira, ${ }^{2}$ Evgeny Nudler, ${ }^{3}$ and \\ Asaph Aharoni ${ }^{1,4}$ \\ ${ }^{1}$ Department of Plant Sciences, Weizmann Institute of \\ Science, Rehovot 76100, Israel; ${ }^{2}$ Department of Life Sciences, \\ Ben Gurion University, Beer Sheva 84105, Israel; ${ }^{3}$ Department \\ of Biochemistry, New York University School of Medicine, \\ New York, New York 10016, USA
}

Riboswitches are natural RNA sensors that affect gene control via their capacity to bind small molecules. Their prevalence in higher eukaryotes is unclear. We discovered a post-transcriptional mechanism in plants that uses a riboswitch to control a metabolic feedback loop through differential processing of the precursor RNA $3^{\prime}$ terminus. When cellular thiamin pyrophosphate (TPP) levels rise, metabolite sensing by the riboswitch located in TPP biosynthesis genes directs formation of an unstable splicing product, and consequently TPP levels drop. When transformed in plants, engineered TPP riboswitches can act autonomously to modulate gene expression. In an evolutionary perspective, a TPP riboswitch is also present in ancient plant taxa, suggesting that this mechanism is active since vascular plants emerged 400 million years ago.

Supplemental material is available at http://www.genesdev.org.

Received June 5, 2007; revised version accepted September 24, 2007.

Riboswitches are regions in mRNA that act as metabolite sensors. These selectively bind small molecule targets such as vitamins, amino acids, and nucleotides through a conserved sensor domain. Upon substrate binding, the conformation of a variable "expression platform" coupled to the sensor domain is changed, and this can affect different modes of gene control including transcription termination, translation initiation, or mRNA processing (Mandal and Breaker 2004; Nudler and Mironov 2004; Coppins et al. 2007). Notably, riboswitches exert their functions without the need for protein cofactors (Mironov et al. 2002; Winkler et al. 2002). In most cases, they act in feedback regulation mechanisms; once the level of an end product in a metabolic pathway rises, riboswitch binding occurs, triggering a repression of gene expression in the same pathway. The substrate specificity of riboswitches is extremely high, allowing them to perform their activity amid the presence of numerous

[Keywords: Thiamine pyrophosphate; riboswitch; alternative splicing; higher eukaryotes; metabolite sensing]

${ }^{4}$ Corresponding author.

E-MAIL asaph.aharoni@weizmann.ac.il; FAX 972-89344181.

Article is online at http://www.genesdev.org/cgi/doi/10.1101/gad.443907. related compounds (Sudarsan et al. 2005). In prokaryotes, genetic control mediated by riboswitches is a prevalent phenomenon, and the dozen riboswitches identified to date regulate $>3 \%$ of all bacterial genes (Nudler 2006).

Thiamin pyrophosphate (TPP) is a coenzyme derived from vitamin B1 (thiamin) that is synthesized by bacteria, fungi, and plants, and serves as a dietary requirement for humans and other animals. It is an obligatory cofactor that plays an important role in amino acid and carbohydrate metabolism; thus, fine control of its levels is essential for the viability of all living cells. TPP-binding riboswitches, first identified in Bacillus subtilis and Escherichia coli (Mironov et al. 2002; Winkler et al. 2002), exist in the genomes of species belonging to most bacterial phyla (Rodionov et al. 2002). Binding of TPP by the riboswitch down-regulates expression of thiamin biosynthesis genes by inducing either the formation of a transcription terminator hairpin or the formation of a Shine-Dalgarno sequester hairpin (Mironov et al. 2002; Rodionov et al. 2002; Winkler et al. 2002). Much less is known about riboswitch-mediated gene control in eukaryotic organisms, and TPP-binding riboswitches are the only ones identified to date (Kubodera et al. 2003; Sudarsan et al. 2003). Very recently, Cheah et al. (2007) demonstrated that a TPP riboswitch controls expression of the THI4 and NMT1 genes in Neurospora crassa by directing the splicing of an intron located in the $5^{\prime}$ untranslated region (UTR). Intron retention results in the appearance of upstream and out-of-frame initiation codons, whereas intron splicing generates a complete and correct open reading frame. While the prokaryotic and fungi riboswitches are located in the 5' UTR, a plant TPP riboswitch located in the 3' UTR of the Arabidopsis THIAMINE C SYNTHASE gene (AtTHIC) was recently identified (Sudarsan et al. 2003). This difference in location suggests a unique mode of action for the plant riboswitch. Yet, the mechanisms mediated by riboswitch activity in higher eukaryotes, particularly in the plant kingdom, remain to be elucidated. Here we propose a unique prevalent model for TPP riboswitch-controlled gene expression in all flowering plants, according to which TPP binding to THIC pre-mRNA engenders alternative splicing that leads to the generation of an unstable transcript, which in turn lowers TPP biosynthesis.

\section{Results and Discussion}

The concentration of TPP controls alternative splicing in the 3' UTR of flowering plant THIC genes

The conservation of the TPP-binding riboswitch in the plant kingdom was examined by computational screening of plant EST libraries (http://compbio.dfci.harvard. edu/tgi). We discovered that a TPP riboswitch is present in the 3' UTR of THIAMINE C SYNTHASE (THIC) genes of 13 different plant species (Supplementary Table S2). Moreover, in eight of them, two alternatively spliced variants were detected in which the second intron of the 3' UTR (int2) was either retained or spliced out, without affecting the coding sequence (Fig. 1A; Supplementary Table S2). In all cases, the $3^{\prime}$ splice site of int2 was located inside the riboswitch P2 box. Previous studies showed that RNA folding at 3' splice sites may alter the 


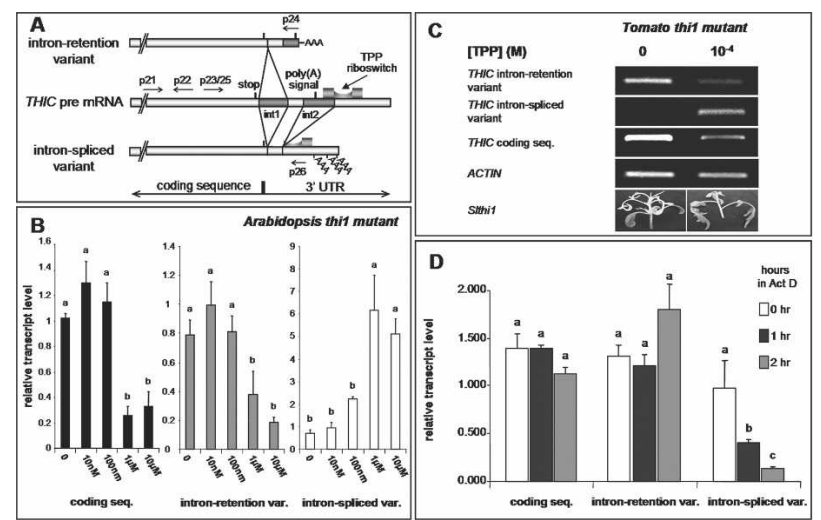

Figure 1. TPP-dependent alternative splicing in the $3^{\prime}$ UTR of Arabidopsis and tomato THIC genes. (A) Schematic representation of the THIC pre-mRNA and the two RNA splicing products, marking the TPP riboswitch, introns 1 and 2 (dark gray), and exons (light gray). Numbers indicate oligonucleotides used for experiments described in $B$ (see Supplemental Material). (B) Transcript levels in TPP-treated Atthi1 mutant seedlings resolved by qPCR experiments $(n=3 ; P<0.01$; SEM; see Materials and Methods for details). (var.) Variant. The oligonucleotides (see A) used were \#21 and \#22 (coding seq.), \#23 and \#24 (intron-retention var.), and \#25 and \#26 (intronspliced var.), and AtACTIN2 was used as endogenous control (oligonucleotides \#34 and \#35). (C) End-point analysis RT-PCR products that monitor the levels of the THIC RNA splicing products in the tomato thiamin auxotroph mutant strain grown under increasing TPP concentrations. The two splicing variants were amplified with specific oligonucleotides (\#8 and \#9, and \#8 and \#10), and the coding region was amplified with oligonucleotides derived from conserved regions (\#8 and \#11). The intron-retention variant, the intron-spliced variant, and the coding sequence were amplified for 31,35 , and 33 cycles, respectively. The effect of TPP addition on whole plants is shown at the bottom of each panel. $(D)$ Stability of the AtTHIC splice variants and coding sequence during variable time points after the addition of the transcription inhibitor actinomycin D to Arabidopsis cell suspension culture supplemented with $1 \mathrm{mM}$ TPP, as resolved by qPCR using the same oligonucleotides as in $B(n=3 ; P<0.01$; SEM). AtUBIQUITINC was used as the endogenous control (oligonucleotides \#27 and \#28). The decay of the intron-spliced variant was on average $63 \%$ per hour.

splicing process (Buratti and Baralle 2004). We therefore predicted that a conformation change due to ligand binding by the riboswitch would alter the splicing of this intron.

To test the prediction that ligand binding affects alternative splicing of THIC, we analyzed the expression of the two RNA splicing products in Arabidopsis (At) plants supplemented with increasing TPP concentrations. When such experiments were conducted with wild-type Arabidopsis plants (containing endogenous TPP), the effect of adding exogenous TPP on AtTHIC splicing was barely detectable (Supplementary Fig. S1B). Therefore, a thiamin auxotroph mutant strain of Arabidopsis (Atthi1) (Supplementary Fig. S1A; Li and Redei 1969) that contains very low levels of endogenous TPP was used. The results showed a fourfold decrease in the expression of the intron-retention variant in the presence of rising TPP concentrations (from 0 to $10 \mu \mathrm{M}$ ), while expression of the intron-spliced variant increased sevenfold (Fig. 1B). Furthermore, testing the expression of the total THIC mRNA population (using oligonucleotides driven from the coding sequence) revealed a 3.75fold decrease with the same rising TPP concentrations (Fig. 1B). For all three transcripts, the statistical signifi- cant change in expression was detected between $100 \mathrm{nM}$ and $1 \mu \mathrm{M}$ TPP (Fig. 1B). This finding is in correspondence with the Kd of the Arabidopsis TPP riboswitch observed in our in-line probing assay ( $500 \mathrm{nM})$ (Fig. 4C, below). In order to assess the universality of the TPP effect on alternative splicing, similar experiments were performed using a tomato (Solanum lycopersicon) thiamin auxotroph mutant (Boynton 1966). Indeed, as detected for AtTHIC, the tomato THIC RNA splicing products showed a TPP concentration-dependent expression pattern (Fig. 1C). These results, from two different plant species, provide evidence that the TPP level determines the pattern of RNA splicing of the THIC precursor mRNA.

The two RNA splicing variants of the THIC gene differ in their stability

Elevated levels of TPP results in the generation of a transcript in which the second intron (int2) of the $3^{\prime}$ UTR is spliced out. We found that the THIC intron-spliced variant is different from the intron-retention variant in two major features. The intron-retention variant has a single consensus polyadenylation [poly(A) formation] signal, and polyadenylation occurs at one accurate site (U370 in the 3' UTR, detected by 3' Rapid Amplification of cDNA Ends [RACE] experiment) (Supplementary Fig. S2). However, in the intron-spliced variant, the consensus polyadenylation signal is eliminated, and polyadenylation occurs at a range of positions along the $3^{\prime}$ UTR (from U531 to C945, detected by 3' RACE experiments). Another feature that distinguishes between the two RNA splicing products is that the $3^{\prime}$ UTR transcript is longer when int 2 is spliced out due to the absence of a consensus poly(A) signal (Fig. 1A; Supplementary Fig. S2). It is documented that the selection of polyadenylation sites and 3' UTR length may result in different intrinsic stabilities of a transcript (Maret et al. 2004). The observation that the overall transcript level of the THIC gene (the sum of RNA splicing products) is reduced while the level of the intron-spliced variant is increased (under elevated TPP concentrations) suggests reduced stability of the intron-spliced variant.

To evaluate the difference in stability between the two RNA splicing products, we examined their decay rate following incubation of Arabidopsis cell suspension cultures supplemented with the transcription inhibitor actinomycin D (Fig. 1D; Supplementary Fig. S1C). The results showed that the relative stability of the intronspliced variant is much lower than that of the intron-retention variant. During the $2 \mathrm{~h}$ following the actinomycin D treatment, the intron-spliced variant exhibited an average decay rate of $63 \%$ per hour, while the intron-retention variant and the overall transcript level of the AtTHIC gene did not show a significant decay (Fig. 1D). The rapid decay of the intron-spliced variant detected here suggests that our analysis of its expression levels (e.g., Fig. 1B), particularly under high TPP concentration, is an underestimation of its genuine production rate.

The results show that the two alternatively spliced transcripts formed as a result of riboswitch activity differ in their stability, the intron-spliced variant retaining a much higher decay rate (compared with the intron-retention variant). 


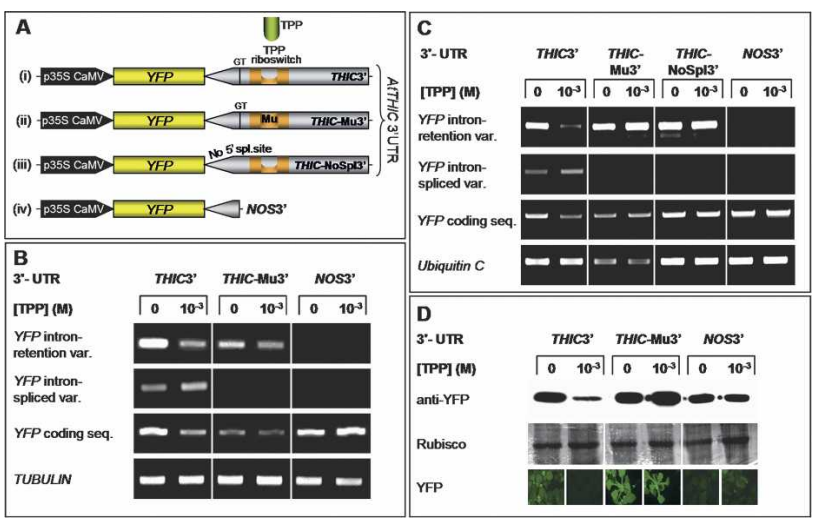

Figure 2. TPP riboswitch-dependent alternative splicing in transgenic Arabidopsis plants. (A) Schematic representation of the constructs used to generate transgenic Arabidopsis plants and protoplasts expressing a YFP reporter gene that was transcriptionally fused to the native AtTHIC 3' UTR (i), the same 3' UTR but with a point mutation disabling riboswitch ligand binding (A515G) (ii), the same 3' UTR but with a point mutation in the $5^{\prime}$ splice (spl.) site of int2 that prevents splicing in this region (iii) (Fig. 1A), and YFP transcriptionally fused to the NOPALINE SYNTHASE (NOS) terminator (iv). (B) RT-PCR end-point analysis experiments in which the transcript levels of the YFP RNA splicing products and the coding region were monitored in transgenic plants grown with or without additional TPP. The intron-retention variant, the intron-spliced variant, and the coding sequence were amplified for 18,22 , and 18 cycles, respectively. (C) RT-PCR end-point analysis experiments in which transcript levels of the YFP RNA splicing products and the coding region were detected in Arabidopsis protoplasts transiently transformed with the corresponding constructs, and incubated with or without additional TPP. The intron-retention variant, the intronspliced variant, and the coding sequence were amplified for 22, 22, and 27 cycles, respectively. (D) YFP expression in transgenic Arabidopsis plants determined by fluorescence and Western blot analyses.

Processing of THIC pre-mRNA is dependent on TPP riboswitch activity, and engineered riboswitches can act autonomously to modulate gene expression

We further investigated whether the alteration in splicing under high TPP levels is independently mediated by the TPP riboswitch activity. These experiments were performed in planta, with the following constructs (Fig. 2A): (i) the YELLOW FLUORESCENCE PROTEIN (YFP) transcriptionally fused to the native AtTHIC 3' UTR (35S:YFP-THIC3'); (ii) the same construct but with a single point mutation (A515G relative to the stop codon) in the $3^{\prime}$ UTR that prevents the riboswitch from binding TPP (35S:YFP-THIC-Mu3') (Sudarsan et al. 2003, 2005; Thore et al. 2006); (iii) the same construct but with a mutated 5' splice site of int2 (Fig. 1A) that prevents splicing of this intron (35S:YFP-THIC-NoSpl3'); and (iv) the same construct but with the YFP fused to the NOPALINE SYNTHASE terminator (35S:YFP-NOS3').

The expression of the two RNA splicing products and the YFP reporter was examined in two plant systems. In the first system, stably transformed Arabidopsis plants harboring the constructs 35S:YFP-THIC3', 35S:YFPTHIC-Mu3', and 35S:YFP-NOS3' were used (Fig. 2B,D). The second system used Arabidopsis protoplasts transiently transformed with all four constructs (Fig. 2C). In every experiment, reporter expression was analyzed with and without supplemental TPP. Remarkably, under high TPP levels, expression of the intron-retention variant in 35S:YFP-THIC3' decreased and that of the intron-spliced variant increased (Fig. 2B,C). No expression of the intron-spliced variant was detected in both systems harboring either the mutated riboswitch (35S:YFP-THICMu3') or the mutated $5^{\prime}$ splice site of int2 (35S:YFPTHIC-NoSpl3'). Expression of the retention variant in transgenic plants harboring the 35S:YFP-THIC-Mu3' was slightly reduced (Fig. 2B), suggesting that the mutation introduced in our construct THIC-Mu3' reduces the riboswitch affinity to TPP but does not abolish its activity completely (Sudarsan et al. 2005). Expression of the retention variant in transgenic plants harboring the 35S:YFP-THIC-NoSpl3' was not affected by additional TPP (Fig. 2C).

We also examined the level of YFP gene expression in the different transformed lines (using oligonucleotides specific to its coding sequence). Addition of TPP reduced the expression of YFP in 35S:YFP-THIC3' plants but had no such effect in plants or protoplasts harboring 35S:YFP-THIC-Mu3' or 35S:YFP-THIC-NoSpl3', as well as in the control 35S:YFP-NOS3' plants (Fig. 2B,C). The fact that disabling splicing of int 2 prevented the decrease in overall YFP expression demonstrated that alternative splicing is essential for riboswitch controlled expression. At the protein level, YFP accumulation (measured either by Western blotting or by YFP fluorescence analysis) was greatly reduced in 35S:YFP-THIC3' plants supplemented with TPP, while its expression was not altered in 35S:YFP-THIC-Mu3' as well as in 35S:YFP-NOS3' (Fig. 2D).

Taken together, these experiments show that when transformed in plants, engineered TPP riboswitch can act autonomously to modulate gene expression. They also demonstrate that alternative splicing in the 3' UTR of the transgene is dependent on riboswitch activity. Furthermore, under high TPP concentration, alternative splicing is essential for the reduction in mRNA levels.

The TPP riboswitch is present in the THIC gene of all plant taxa, and an additional TPP riboswitch, lost during gymnosperm evolution, is present in the THI1 gene of ancient plants

To trace the evolutionary origin of plant riboswitches, we searched for homologous sequences of the TPP riboswitch across the plant kingdom. Homologous sequences were identified in the THIC 3' UTR of all ex-

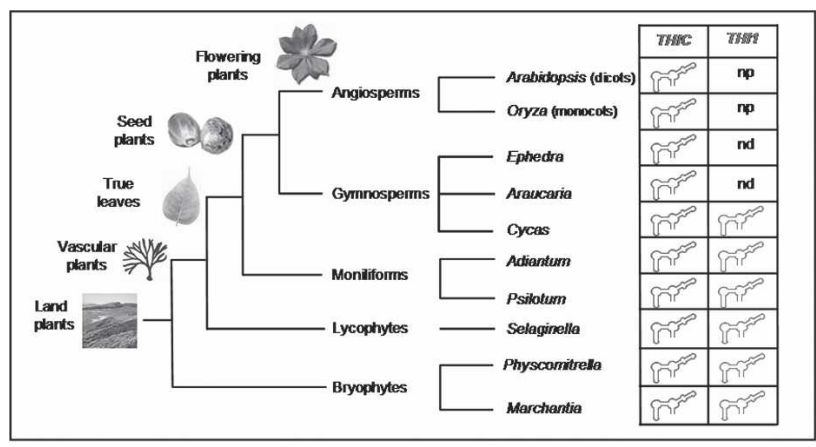

Figure 3. The TPP riboswitch is present in two genes of the thiamin biosynthesis pathway in ancient plants. The presence of the TPP riboswitch in the $3^{\prime}$ UTR of the THIC and THI1 genes across the plant kingdom is depicted. (np) Not present, based on analysis of whole genome sequences; (nd) not detected in PCR assays. 


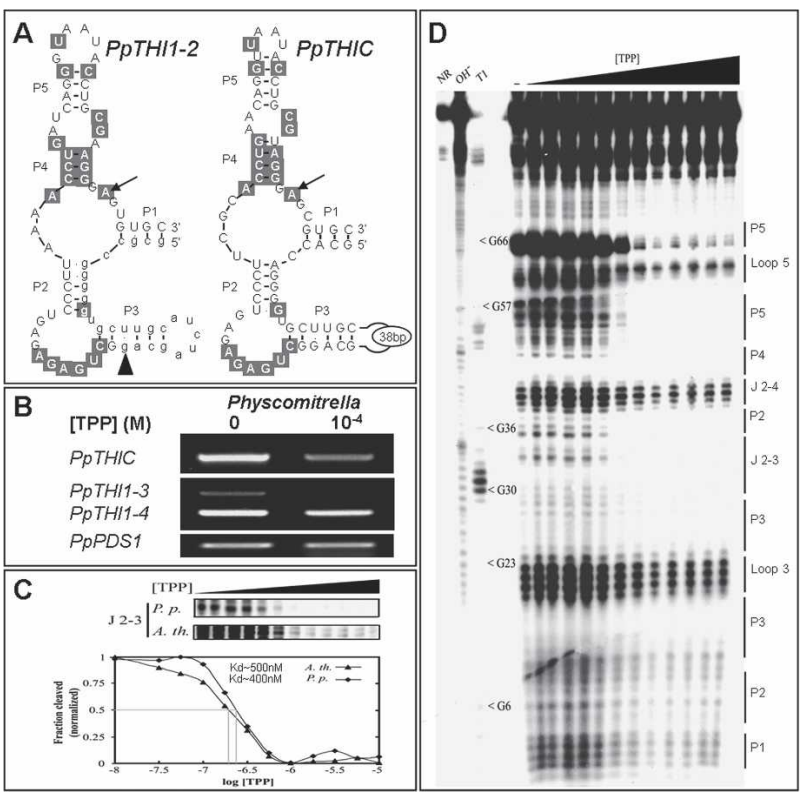

Figure 4. (A) Schematic representations of the TPP-binding domain of $P$. patens $(P p)$ located in the $3^{\prime}$ UTRs of the PpTHIC and PpTHI1-2 genes. Exon and intron sequences are indicated by uppercase and lowercase letters, respectively. Arrows indicate the adenine base that forms the crucial assembly platform of the TPP riboswitch (Serganov et al. 2006; Thore et al. 2006); the arrowhead marks the 3 splice site. Shadowed nucleotides are mostly conserved among TPP riboswitches (Sudarsan et al. 2003). (B) RT-PCR end-point analysis products that monitor the transcript levels of the PpTHIC and PpTHI1 genes in $P$. patens $(P p)$ protomemata grown without or with additional TPP. (PDS) Phytoene desaturase transcript was used for calibration. PpTHIC and PpTHI1 genes were amplified for 25 cycles. (C) Apparent Kd for TPP binding by the TPP riboswitch of Arabidopsis (AtTHIC) and P. patens (PpTHI1-2), determined by in-line probing (Sudarsan et al. 2003). (D) Fragmentation pattern of the in vitro-transcribed 80-bp RNA molecule that comprises the P. patens THI1-2 TPP-riboswitch. The in-line probing assay was performed in the presence of increasing TPP concentrations $(0-10 \mu M)$. (NR) Nonreacted RNA; $\left(\mathrm{OH}^{-}\right)$RNA subjected to alkaline degradation; (T1) RNA submitted to RNase T1 digestion.

amined species, ranging from the liverwort Marchantia polymorpha (bryophytes) to higher plants (angiosperms) (Fig. 3; Supplementary Fig. S3). However, when we examined the THIC gene of Physcomitrella patens ( $P$. patens or $P p$ ), we could not find evidence for splicing in the second intron of its $3^{\prime}$ UTR, as detected for flowering plants (int2) (see Fig. 1A). Therefore, it seems that the PpTHIC gene is regulated by a different mechanism than the one used in flowering plants, which does not involve alternative splicing in its $3^{\prime}$ UTR. This is in accordance with previous studies reporting that intron-containing genes and genes undergoing alternative splicing have increased considerably from ancestral to higher eukaryotes and that alternative splicing is a relatively advanced evolutionary process (Reddy 2007).

TPP biosynthesis in plants occurs by the condensation of thiazol and pyrimidine moieties (Supplementary Fig. S4). Interestingly, we also found that in $P$. patens, a putative TPP riboswitch sequence is present in the $3^{\prime}$ UTR of the THIAZOL BIOSYNTHESIS 1 (THI1; a THI4 ortholog) gene family members, which encode a thiazol biosynthetic enzyme (Fig. 4A; Supplementary Fig. S5). This suggests that the TPP biosynthesis pathway is controlled by a system of double riboswitch check points. In contrast to Arabidopsis, there are four THI1 genes in $P$. patens, and three of them possess a TPP riboswitch in the 3' UTR (PpTHI1-2, PpTHI1-3, PpTHI1-4). In two of these genes (PpTHI1-2, PpTHI1-3), splicing at the $3^{\prime}$ UTR occurs, and the intron $3^{\prime}$ splice site is located at the P3 box of the TPP riboswitch. However, this intron seems to be constitutively spliced as we could not find, either experimentally or in public databases, evidence for the presence of an intron-retention variant (Fig. 4B; Supplementary Fig. S5). In contrast, no indication of splicing was evident in the 3' UTR of the third $P$. patens gene (PpTHI1-4) (Supplementary Fig. S5). PpTHIC, PpTHI1-3, and PpTHI1-4 are down-regulated upon TPP supplementation (expression of PpTHI1-1 and PpTHI1-2 was barely detectable) (Fig. 4B). In-line probing analysis of the PpTHI1 80-base-pair (bp) TPP riboswitch revealed that it exhibits a TPP-dependent structural modulation that is comparable with the parallel bacterial and Arabidopsis TPP riboswitches (Fig. 4A,D; Sudarsan et al. 2003). The PpTHI1 RNA binds TPP at a $\mathrm{K}_{\mathrm{D}}$ of $\sim 400 \mathrm{nM}$ (Fig. $4 \mathrm{C})$, which is comparable with the $\mathrm{K}_{\mathrm{D}}$ value ( 500 $\mathrm{nM}$ ) obtained by our in-line probing assay using the AtTHIC riboswitch.

In an evolutionary perspective, we found that an additional TPP riboswitch is present in the THI1 gene of ancient plant taxa (bryophytes, lycophytes, and moniliforms), including in the most ancient land plants, the liverwort Marchantia polymorpha, but it was lost from this gene family during gymnosperm evolution (Fig. 3; Supplementary Fig. S3). We also discovered that alternative splicing in the 3' UTR of the THIC and THI1 genes occurred already in lycophytes (i.e., Selaginella), an ancient vascular plant family that appeared 150-200 million years before angiosperms such as Arabidopsis and rice (Fig. 3; Supplementary Fig. S3).

In this study, we report on a novel post-transcriptional regulatory mechanism for gene regulation in plants (Fig. 5). The findings reveal that a TPP riboswitch mediates feedback regulation in the thiamin biosynthesis pathway through modulation of alternative splicing in the $3^{\prime}$ UTR region of a gene of this pathway. High levels of TPP in the cell promote ligand binding and a change in the riboswitch structural conformation. Consequently, an unstable intron-spliced variant is formed, which in turn results in a decrease of TPP levels. Alternatively, under

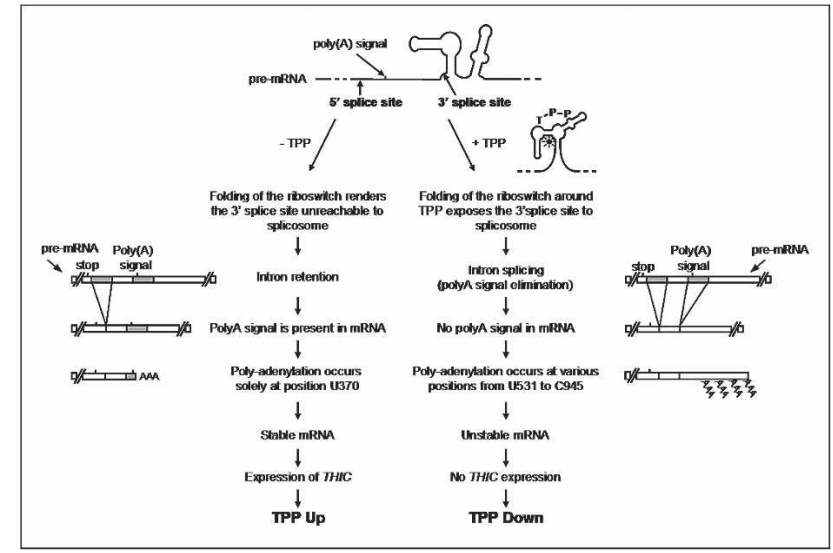

Figure 5. A model for TPP riboswitch-mediated regulation of gene expression via alternative splicing in vascular plants. The $3^{\prime}$ splice site is highlighted with a star. 
low cellular TPP levels, the conformation of the riboswitch dictates intron retention that generates a stable transcript, which in turn promotes an increase in TPP biosynthesis. An intriguing question that arises is: How does riboswitch conformational change direct alternative splicing? The structure of the Arabidopsis riboswitch-TPP complex was recently resolved by Thore et al. (2006). Examination of this complex structure reveals that in the ligand-bound state, the $3^{\prime}$ splice site of the intron is exposed (Supplementary Fig. S6). We therefore hypothesize that ligand binding brings about conformational changes in the $3^{\prime}$ region of the intron that promote splicing. On the other hand, riboswitch conformation in the unbound state could therefore hinder intron processing by preventing the splicing machinery from accessing this region (Buratti and Baralle 2004).

Riboswitch-mediated alternative splicing gene expression control is active in both ancient and modern vascular plants and hence might have appeared during their emergence 400 million years ago. The riboswitch loss in the thiazol branch of the thiamin pathway (in THI1) in the gymnosperm lineage suggests that a single point of riboswitch control may be sufficient, especially if new regulatory mechanisms have been adopted in parallel. The data reported here on the riboswitch-mediated control of both endogenous and foreign genes expression revealed a unique regulatory mechanism that acts autonomously in planta, independent of promoter activation or effects exerted by coding sequences or the $5^{\prime}$ UTR. To what extent riboswitch-dependent processes are widespread in plants is hard to predict at this point, but we expect that this report will trigger additional findings of a similar nature. This study thus paves the way for future engineering of plant riboswitches, in an attempt to map metabolite concentrations and regulate pre-mRNA splicing of genes that are involved in their biosynthetic pathways.

\section{Materials and methods}

Oligonucleotides

All oligonucleotides used in our experiments are listed in Supplementary Table S1.

Plant material

See the Supplemental Material for all details regarding plant material.

Plant transformation

For the generation of transgenic Arabidopsis, the AtTHIC 3' UTR was amplified by PCR using Arabidopsis genomic DNA (ColO) with oligonucleotides \#1 and \#2 (Supplementary Table S1). The mutation (A515G, starting from the stop codon) was introduced using the megaprimerbased mutagenesis strategy (Kammann et al. 1989) with oligonucleotides $\# 1$, \#2, and \#20. The AtTHIC 3' UTR was then fused to the YFP reporter gene, and the fusion fragment was subsequently inserted downstream from the double $35 \mathrm{~S}$ promoter of the Cauliflower Mosaic Virus (CaMV). The $5^{\prime}$ splice site mutation was obtained by amplifying the cassettecontaining plasmid with oligonucleotides \#29 and \#30, followed by selfligation of the PCR product and transformation of the resulting plasmid into Escherichia coli. This resulted in one construct containing the following mutations: T263A, T267A, T276A, T279A, T287A, and T292A. The cassettes were then inserted into the pBinPlus binary vector containing the kanamycin resistance gene for the selection of transformants (van Engelen et al. 1995). Arabidopsis plants were transformed using the floral-dip method (Clough and Bent 1998), and kanamycin-resistant seedlings were then transferred to soil.

Molecular biology and microscopy

Unless specified, all molecular biology manipulations were performed as described in Sambrook et al. (1989). RNA extractions were all performed using the RNeasy kit (Qiagen) and DNA extractions were performed using the CTAB method (Doyle and Doyle 1987).

Quantitative real-time PCR (qPCR) gene expression analysis was performed with three biological replicates using gene/variant-specific qPCR oligonucleotide pairs, designed with Primer Express software (Applied Biosystems). Specific oligonucleotides sequences are provided in Supplementary Table S1 (\#21-\#26), and their efficiency standard curves are depicted in Supplementary Figure S1D. AtACTIN2 was used as an endogenous control for the expression analysis of each AtTHIC variant, and AtUBIQUITIN $C$ was used as an endogenous control for the relative stability experiment (with oligonucleotides \#34-\#35 and \#27-\#28, respectively). A fixed amount of DNase-treated total RNA was reversetranscribed using AMV Reverse Transcriptase (EurX Ltd.). RT-PCR reactions were tracked on an ABI 7300 instrument (Applied Biosystems) using the PlatinumR SYBR SuperMix (Invitrogen). Each sample was PCR-amplified from the same amount of cDNA template in triplicate reactions. Following an initial step in the thermal cycler for $15 \mathrm{~min}$ at $95^{\circ} \mathrm{C}, \mathrm{PCR}$ amplification proceeded for 45 cycles of $15 \mathrm{sec}$ at $95^{\circ} \mathrm{C}$ and 30 sec at $60^{\circ} \mathrm{C}$, and was completed by melting curve analysis to confirm specificity of the PCR products. The baseline and threshold values were adjusted according to the manufacturers' instructions.

RT-PCR end-point analysis was performed as described in Aharoni et al. (2004a) with oligonucleotides \#3-\#7 (Supplementary Table S1). In tomato, the intron-retention variant was detected with oligonucleotides \#8 and \#9, the intron-spliced variant was detected with \#8 and \#10, and the SITHIC coding region transcripts were detected with \#8 and \#11 (Supplementary Table S1). The TUBULIN or UBIQUITIN C genes (for Arabidopsis) and ACTIN gene (for tomato) were used for calibration with oligonucleotides \#17-\#18, \#28-\#33, and \#12-\#13, respectively. The YFP mRNA transcript levels were determined using oligonucleotides \#16 and $\# 4$ for the intron-retention variant and \#16 and \#5 for the intron-spliced variant, and the YFP-coding region transcripts were determined with \#14 and \#15. For all other plant species, DNA or RNA fragments corresponding to the $3^{\prime}$ region of the THIC and THI1 genes were amplified with the forward oligonucleotides \#8 and \#10, respectively, and reverse \#9. All splicing products were confirmed by sequencing.

For RNA stability determination, actinomycin D (Sigma, catalog no. A1410) was added to Arabidopsis suspension cell culture to a final concentration of $50 \mu \mathrm{g} / \mathrm{mL}$. Cells supplemented with $1 \mathrm{mM}$ TPP were harvested at three or four time points $(0,1 \mathrm{~h}, 2 \mathrm{~h}, 4 \mathrm{~h})$, followed by RNA extraction and quantitative real-time experiments with the specific oligonucleotides \#21-\#28 as described above (Fig. 1D). RNA stability was also determined by RT-PCR end-point analysis with the variant-specific oligonucleotides (\#3-\#7) (Supplementary Fig. S1C). All splicing products were confirmed by sequencing. 3' RACE experiments were performed as described by Aharoni et al. (2004b), with oligonucleotides \#8, \#19, \#31, or \#32. The YFP signal was detected by a standard fluorescence microscope.

In-line probing

The in-line probing experiment was conducted as described previously by Sudarsan et al. (2003). Briefly, RNA was synthesized in vitro from DNA templates by $\mathrm{T} 7$ polymerase, $5^{\prime}$-end-labeled with ${ }^{32} \mathrm{P}$, and gel-purified. The radiolabeled RNA was subjected to in-line probing by incubation for $40 \mathrm{~h}$ at $25^{\circ} \mathrm{C}$ in $20 \mathrm{mM} \mathrm{MgCl}, 100 \mathrm{mM} \mathrm{KCl}$, and $50 \mathrm{mM}$ Tris. $\mathrm{HCl} / \mathrm{pH}$ 8.31 , in the presence of increasing TPP concentrations from 0 to $10 \mu \mathrm{M}$. The partially cleaved fragments were resolved on a denaturing gel $(8 \mathrm{M}$ urea- $8 \%$ polyacrylamide), which was further subjected to a PhosphorImager analysis. Kd values were estimated as described in Cheah et al. (2007).

\section{Acknowledgments}

We acknowledge Gad Galili for helpful discussions, Idan Gabdank and Miriam Ben-David for their help with in-line probing, Simcha Lev-Yadun and the Botanic Garden of Tel Aviv University for plant material, Dvora Dolev for Arabidopsis cell suspension cultures, Tzahi Arazi for providing Physcomitrella, and Assaf Zemach for help with protoplast preparation. Michal Shapira is an incumbent of the Thaler Chair for Plant Genetics at BGU. Plant research in the Shapira laboratory is supported by the Israel Science Foundation and by the Faculty of Natural Sciences of BGU. Asaph Aharoni is an incumbent of the Adolfo and Evelyn Blum Career Development Chair at the Weizmann Institute. The work in the Aharoni laboratory was supported by the William Z. and Eda Bess Novick New Scientists Fund and the Henry S. and Anne Reich Family Foundation. 


\section{References}

Aharoni, A., Dixit, S., Jetter, R., Thoenes, E., van Arkel, G., and Pereira, A. 2004a. The SHINE clade of AP2 domain transcription factors activates wax biosynthesis, alters cuticle properties, and confers drought tolerance when overexpressed in Arabidopsis. Plant Cell 16: 2463-2480.

Aharoni, A., Giri, A.P., Verstappen, F.W., Bertea, C.M., Sevenier, R., Sun, Z., Jongsma, M.A., Schwab, W., and Bouwmeester, H.J. 2004b. Gain and loss of fruit flavor compounds produced by wild and cultivated strawberry species. Plant Cell 16: 3110-3131.

Boynton, J.E. 1966. Chlorophyll-deficient mutants in tomato requiring vitamin B1. I. Genetics and physiology. Hereditas 56: 171-199.

Buratti, E. and Baralle, F.E. 2004. Influence of RNA secondary structure on the pre-mRNA splicing process. Mol. Cell. Biol. 24: 10505-10514.

Cheah, M.T., Wachter, A., Sudarsan, N., and Breaker, R.R. 2007. Control of alternative RNA splicing and gene expression by eukaryotic riboswitches. Nature 447: 497-500.

Clough, S.J. and Bent, A.F. 1998. Floral dip: A simplified method for Agrobacterium-mediated transformation of Arabidopsis thaliana. Plant I. 16: 735-743.

Coppins, R.L., Hall, K.B., and Groisman, E.A. 2007. The intricate world of riboswitches. Curr. Opin. Microbiol. 10: 176-181.

Doyle, J. and Doyle, J. 1987. A rapid DNA isolation procedure from small quantities of fresh leaf tissues. Phytochem. Bull. 19: 11-15.

Kammann, M., Laufs, J., Schell, J., and Gronenborn, B. 1989. Rapid insertional mutagenesis of DNA by polymerase chain reaction (PCR). Nucleic Acids Res. 17: 5404.

Kubodera, T., Watanabe, M., Yoshiuchi, K., Yamashita, N., Nishimura, A., Nakai, S., Gomi, K., and Hanamoto, H. 2003. Thiamine-regulated gene expression of Aspergillus oryzae thiA requires splicing of the intron containing a riboswitch-like domain in the $5^{\prime}$-UTR. FEBS Lett. 555: $516-520$.

Li, S.L. and Redei, G.P. 1969. Thiamine mutants of the crucifer, Arabidopsis. Biochem. Genet. 3: 163-170.

Mandal, M. and Breaker, R.R. 2004. Adenine riboswitches and gene activation by disruption of a transcription terminator. Nat. Struct. Mol. Biol. 11: 29-35.

Maret, D., Boffa, M.B., Brien, D.F., Nesheim, M.E., and Koschinsky, M.L. 2004. Role of mRNA transcript stability in modulation of expression of the gene encoding thrombin activable fibrinolysis inhibitor. I. Thromb. Haemost. 2: 1969-1979.

Mironov, A.S., Gusarov, I., Rafikov, R., Lopez, L.E., Shatalin, K., Kreneva, R.A., Perumov, D.A., and Nudler, E. 2002. Sensing small molecules by nascent RNA: A mechanism to control transcription in bacteria. Cell 111: 747-756.

Nudler, E. 2006. Flipping riboswitches. Cell 126: 19-22.

Nudler, E. and Mironov, A.S. 2004. The riboswitch control of bacterial metabolism. Trends Biochem. Sci. 29: 11-17.

Reddy, A.S.N. 2007. Alternative splicing of pre-messenger RNAs in plants in the genomic era. Annu. Rev. Plant Biol. 58: 267-294.

Rodionov, D.A., Vitreschak, A.G., Mironov, A.A., and Gelfand, M.S. 2002. Comparative genomics of thiamin biosynthesis in procaryotes. New genes and regulatory mechanisms. J. Biol. Chem. 277: 4894948959.

Sambrook, J., Fritsch, E., and Maniatis, T. 1989. Molecular cloning: A laboratory manual. Cold Spring Harbor Laboratory Press, Cold Spring Harbor, NY.

Serganov, A., Polonskaia, A., Phan, A.T., Breaker, R.R., and Patel, D.J. 2006. Structural basis for gene regulation by a thiamine pyrophosphate-sensing riboswitch. Nature 441: 1167-1171.

Sudarsan, N., Barrick, J.E., and Breaker, R.R. 2003. Metabolite-binding RNA domains are present in the genes of eukaryotes. RNA 9: 644647.

Sudarsan, N., Cohen-Chalamish, S., Nakamura, S., Emilsson, G.M., and Breaker, R.R. 2005. Thiamine pyrophosphate riboswitches are targets for the antimicrobial compound pyrithiamine. Chem. Biol. 12: 13251335.

Thore, S., Leibundgut, M., and Ban, N. 2006. Structure of the eukaryotic thiamine pyrophosphate riboswitch with its regulatory ligand. Science 312: 1208-1211.

van Engelen, F.A., Molthoff, J.W., Conner, A.J., Nap, J.P., Pereira, A., and Stiekema, W.J. 1995. pBinPlus: An improved plant transformation vector based on pBIN19. Transgenic Res. 4: 288-290.

Winkler, W., Nahvi, A., and Breaker, R.R. 2002. Thiamine derivatives bind messenger RNAs directly to regulate bacterial gene expression. Nature 419: 952-956. 


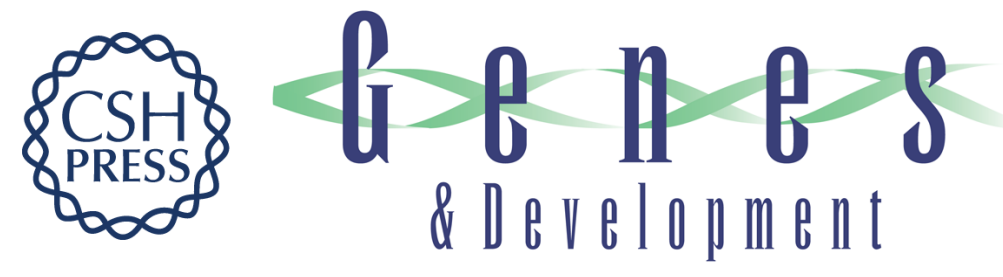

\section{Riboswitch-dependent gene regulation and its evolution in the plant kingdom}

Samuel Bocobza, Avital Adato, Tali Mandel, et al.

Genes Dev. 2007, 21:

Access the most recent version at doi:10.1101/gad.443907

Supplemental http://genesdev.cshlp.org/content/suppl/2007/11/01/21.22.2874.DC1
Material

References This article cites 24 articles, 6 of which can be accessed free at:

http://genesdev.cshlp.org/content/21/22/2874.full.html\#ref-list-1

License

Email Alerting

Receive free email alerts when new articles cite this article - sign up in the box at the top

Service

right corner of the article or click here.

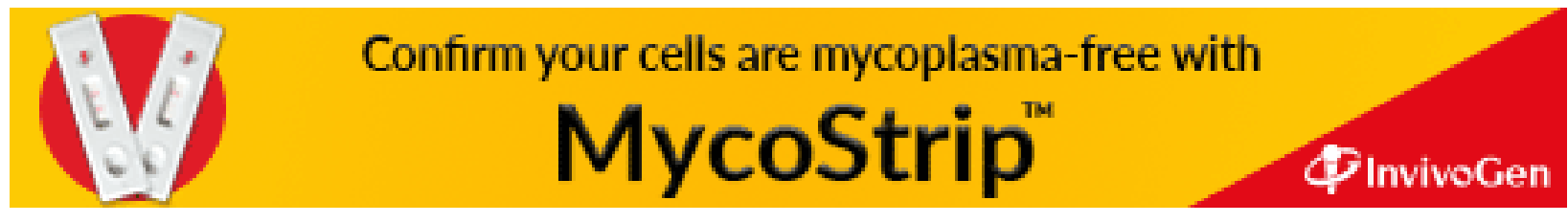

